\title{
Cost Thirty-day readmission and reoperation after surgery for spinal tumors: a National Surgical Quality Improvement Program analysis
}

\author{
Aditya V. Karhade, BE, Viren S. Vasudeva, MD, Hormuzdiyar H. Dasenbrock, MD, Yi Lu, MD, PhD, \\ William B. Gormley, MD, MPH, MBA, Michael W. Groff, MD, John H. Chi, MD, MPH, and \\ Timothy R. Smith, MD, PhD, MPH
}

Cushing Neurosurgery Outcomes Center, Department of Neurosurgery, Brigham and Women's Hospital, Harvard Medical School, Boston, Massachusetts

OBJECTIVE The goal of this study was to use a large national registry to evaluate the 30-day cumulative incidence and predictors of adverse events, readmissions, and reoperations after surgery for primary and secondary spinal tumors.

METHODS Data from adult patients who underwent surgery for spinal tumors (2011-2014) were extracted from the prospective National Surgical Quality Improvement Program (NSQIP) registry. Multivariable logistic regression was used to evaluate predictors of reoperation, readmission, and major complications (death, neurological, cardiopulmonary, venous thromboembolism [VTE], surgical site infection [SSI], and sepsis). Variables screened included patient age, sex, tumor location, American Society of Anesthesiologists (ASA) physical classification, preoperative functional status, comorbidities, preoperative laboratory values, case urgency, and operative time. Additional variables that were evaluated when analyzing readmission included complications during the surgical hospitalization, hospital length of stay (LOS), and discharge disposition.

RESULTS Among the 2207 patients evaluated, $51.4 \%$ had extradural tumors, $36.4 \%$ had intradural extramedullary tumors, and $12.3 \%$ had intramedullary tumors. By spinal level, $20.7 \%$ were cervical lesions, $47.4 \%$ were thoracic lesions, $29.1 \%$ were lumbar lesions, and $2.8 \%$ were sacral lesions. Readmission occurred in $10.2 \%$ of patients at a median of 18 days (interquartile range [IQR] 12-23 days); the most common reasons for readmission were SSIs (23.7\%), systemic infections (17.8\%), VTE (12.7\%), and CNS complications (11.9\%). Predictors of readmission were comorbidities (dyspnea, hypertension, and anemia), disseminated cancer, preoperative steroid use, and an extended hospitalization. Reoperation occurred in $5.3 \%$ of patients at a median of 13 days (IQR 8-20 days) postoperatively and was associated with preoperative steroid use and ASA Class 4-5 designation. Major complications occurred in $14.4 \%$ of patients: the most common complications and their median time to occurrence were VTE (4.5\%) at 9 days (IQR 4-19 days) postoperatively, SSIs $(3.6 \%)$ at 18 days (IQR $14-25$ days), and sepsis (2.9\%) at 13 days (IQR 7-21 days). Predictors of major complications included dependent functional status, emergency case status, male sex, comorbidities (dyspnea, bleeding disorders, preoperative systemic inflammatory response syndrome, preoperative leukocytosis), and ASA Class $3-5$ designation ( $p$ $<0.05$ ). The median hospital LOS was 5 days (IQR 3-9 days), the 30 -day mortality rate was $3.3 \%$, and the median time to death was 20 days (IQR $12.5-26$ days).

CONCLUSIONS In this NSQIP analysis, $10.2 \%$ of patients undergoing surgery for spinal tumors were readmitted within 30 days, $5.3 \%$ underwent a reoperation, and $14.4 \%$ experienced a major complication. The most common complications were SSIs, systemic infections, and VTE, which often occurred late (after discharge from the surgical hospitalization). Patients were primarily readmitted for new complications that developed following discharge rather than exacerbation of complications from the surgical hospital stay. The strongest predictors of adverse events were comorbidities, preopera-

\footnotetext{
ABBREVIATIONS ACS $=$ American College of Surgeons; $A S A=$ American Society of Anesthesiologists; $A U R O C=$ area under the receiver operating curve; $B M I=$ body mass index; $\mathrm{Cl}=$ confidence interval; $\mathrm{COPD}=$ chronic obstructive pulmonary disease; $\mathrm{CPT}=$ Current Procedural Terminology; $\mathrm{Gl}=$ gastrointestinal; $\mathrm{GU}=$ genitourinary; ICD-9-CM = International Classification of Diseases, Ninth Revision, Clinical Modification; INR = international normalized ratio; IQR = interquartile range; LOS = length of stay; NSQIP = National Surgical Quality Improvement Program; PTT = partial thromboplastin time; SIRS = systemic inflammatory response syndrome; SSI = surgical site infection; UTI = urinary tract infection; VTE = venous thromboembolism; $\mathrm{WBC}=$ white blood cell.
}

SUBMITTED April 1, 2016. ACCEPTED May 23, 2016.

INCLUDE WHEN CITING DOI: 10.3171/2016.5.FOCUS16168. 
tive steroid use, and higher ASA classification. These models can be used by surgeons to risk-stratify patients preoperatively and identify those who may benefit from increased surveillance following hospital discharge.

http://thejns.org/doi/abs/10.3171/2016.5.FOCUS16168

KEY WORDS readmission; reoperation; spinal tumor; surgical site infection; venous thromboembolism; complication; metastases

$\mathrm{S}$ PINAL tumors are a major cause of morbidity and mortality among oncology patients. The incidence of tumors of the spinal column is estimated to be 0.62 per 100,000 individuals in the US. ${ }^{33,85}$ Metastatic spinal tumors make up the majority of spinal tumors and are found in as many as $70 \%$ of cancer patients..$^{48,52,65,74,86,93,98}$ In addition to classification by histology, spinal tumors are generally stratified by location as extradural, intradural extramedullary, or intramedullary. The goals of surgical treatment of spinal tumors are to improve functional - particularly ambulatory - status, decrease pain, and in some patients with isolated metastases or primary tumors, improve survival. 1,13,14,16,17,26,27,30,31,38,63,93,95-98 However, surgical approaches to spinal tumors often necessitate extensive reconstruction of the spinal column and may require adjuvant chemotherapy or radiation; therefore, perioperative complication rates after spinal tumor surgery are higher than in other spinal operations. ${ }^{34-36,46-48,50,52,65,67,68,78,80,81,86-88,95,100}$

It is especially important to understand the incidence and predictive factors for adverse events in spinal oncology patients, as a single complication may be associated with morbidity, mortality, and costs. $1,31,37,46,47,52,62,70,72,79,86,95$ Risk stratification of patients preoperatively is important to optimize outcomes, ${ }^{48,52,65,74,86,93,98}$ and prior studies have identified anatomical location, tumor size, tumor histology, extent of resection, surgical approaches, age, functional status, comorbidities, preoperative medication (steroid use, morphine), longer operative time, insurance status, early postoperative complications, and adjuvant chemotherapy and radiotherapy as factors associated with postoperative morbidity and mortality. $1,18-24,39,41,42,44,48,51,52,56,58,66$, 69,78,92,99 Nevertheless, many of these previous publications were retrospective, institutional studies, ${ }^{10,11,55,60,65,87,95}$ and additional prospective data are needed.

The American College of Surgeons-National Surgical Quality Improvement Program (ACS-NSQIP) is a large, prospectively collected registry that collects data on 30day postoperative outcomes from patients undergoing surgical intervention at 517 institutions across the US.,7, 9,49,59,60,61,71,77,90,91 Despite its utility in evaluating perioperative complications and adverse outcomes, few studies have used the NSQIP to analyze the outcomes of patients undergoing surgery for spinal tumors. The goal of this study was to evaluate the 30-day cumulative incidence, time to event, and predictors of major complications, unplanned reoperations, and readmissions after surgery for primary and secondary spinal tumors.

\section{Methods}

\section{Data Source}

The prospective NSQIP registry was used from the years 2011 to 2014. Maintained by the ACS, trained sur- gical reviewers prospectively collect data for the NSQIP using a uniform protocol. Patients enrolled in the NSQIP are followed for 30 days postoperatively, and all complications (among those collected in the NSQIP algorithm) that occurred during this period are recorded; there is no administrative censoring or loss to follow-up of patients entered. The accuracy of data collection by the NSQIP (including cumulative incidence and reasons for readmission) has been externally validated ${ }^{84}$ and this data source has been previously used to evaluate patients undergoing neurosurgical intervention, $4,8,12,14,15,28,29,45,54,83$ including spine surgery. $2,6,9,10,25,40,53,59,61,82,91$ Our institutional review board has exempted the de-identified NSQIP data set from individual review.

\section{Inclusion and Exclusion Criteria}

Patients were included who met the following criteria: 1) were aged 18 years or greater; 2) had a Current Procedural Terminology (CPT) code indicating they underwent laminectomy for excision or biopsy of an extradural (63275-8), intradural extramedullary (63280-3) or intramedullary (63285-7) tumor or they underwent corpectomy for excision of an intraspinal lesion (CPT code 63300-7) and had an International Classification of Diseases, Ninth Revision, Clinical Modification (ICD-9-CM) code indicating a spinal neoplasm $(170.2,170.6,198.3-5$, $213.2,213.6)$; 3) the attending surgeon was a neurosurgeon or an orthopedic surgeon; and 4) the procedure was performed under general anesthesia.

\section{Covariates}

Pertinent covariates collected by the NSQIP were extracted. Age was examined categorically by quartile. Tumor location was evaluated as extradural, intradural extramedullary, or intramedullary. Additionally, location was stratified by involvement of the cervical, thoracic, lumbar, or sacral spine. Sex, race/ethnicity, American Society of Anesthesiologists (ASA) physical classification (1-2, 3, 4-5, or missing), and preoperative functional status (dependent vs independent) were assessed.

Comorbidities collected by the NSQIP-present in at least 20 patients, and missing in less than $10 \%$ of patientswere evaluated: smoking (within the past year), hypertension requiring medication, chronic obstructive pulmonary disease (COPD), dyspnea, diabetes mellitus, and a bleeding disorder (defined by the NSQIP as vitamin K deficiencies, hemophilias, thrombocytopenia, and long-term anticoagulant usage), and unintentional weight loss of more than $10 \%$ within 6 months. Body mass index (BMI) was determined based on height and weight, with obesity defined using WHO criteria: Class I obesity as $30-34.99 \mathrm{~kg} /$ $\mathrm{m}^{2}$ and Class II or III as $>35 \mathrm{~kg} / \mathrm{m}^{2}$. Disseminated cancer is defined by the NSQIP as multiple metastases indicat- 
ing that the cancer is widespread. Pertinent preoperative laboratory values were extracted and stratified: sodium (by $135 \mathrm{mEq} / \mathrm{L}$ ), creatinine (by $1.4 \mathrm{mg} / \mathrm{dl}$ ), albumin (by $3.5 \mathrm{~g} / \mathrm{dl}$ ), white blood cell (WBC) count (by 4000/ul and $12,000 / \mu \mathrm{l}$ ), hematocrit (by 36\%), international normalized ratio (INR; by 1.4), and partial thromboplastin time (PTT; by 40 seconds). Preoperative steroid use (within 30 days) was also extracted. Although data were missing on both recent chemotherapy (within 30 days) and radiation treatment (within 90 days), they were evaluated given the clinical relevance of these variables in oncology patients. An open wound is defined by the NSQIP as any incision that directly communicates with air. Preoperative systemic inflammatory response syndrome (SIRS) is indicated in any patient with at least 2 of the following criteria: temperature greater than $38^{\circ} \mathrm{C}$ or less than $36^{\circ} \mathrm{C}$, heart rate greater than 90 beats per minute, respiratory rate greater than 20 breaths per minute, leukocytosis or leukopenia (WBC count $>12,000 / \mu l$ and $<4000 / \mu l$, respectively), or anion gap acidosis. Preoperative transfusion of greater than 4 units of packed red blood cells in the 72 hours preoperatively is also recorded by the NSQIP. Admission type, preoperative intubation (within 48 hours), case urgency (emergency or elective), and operative time (by quartile) were also extracted. Wound classification compared Class I (clean) operations to Class II (clean-contaminated), III (contaminated), and IV (dirty) designations.

\section{Outcomes}

The outcomes evaluated were major complications, unplanned reoperation, and unplanned readmission. A major complication was defined according to a published categorization as: death; an intracranial (new postoperative coma or stroke), cardiac (cardiac arrest or myocardial infarction), pulmonary (reintubation or prolonged postoperative mechanical ventilation), or renal (acute renal failure) complication; symptomatic venous thromboembolism (VTE); surgical site infection (SSI); sepsis; or reoperation. ${ }^{57}$ Additional minor complications extracted were pneumonia, urinary tract infections (UTIs), and perioperative packed red blood cell transfusions.

Unplanned reoperation (within 30 days) was available for patients from the 2012-2014 years of the NSQIP, and therefore only patients from these years were evaluated for this outcome. The CPT codes associated with the reoperations were also extracted.

Finally, an unplanned hospital readmission, which the NSQIP collects regardless of whether the admission is to the same or to a different facility, was examined. Only unplanned readmissions were evaluated because some patients may require early planned readmission for oncological treatment. Additionally, the NSQIP began collecting data on the primary admission diagnosis associated with the readmission in 2012, some of which (including SSIs, VTE events, and systemic infections) are encoded directly, and others of which are designated through ICD-9-CM codes. When evaluating predictors of unplanned readmission, complications during the surgical hospitalization, length of the index hospitalization, and discharge disposition were also evaluated. Readmission was only assessed among patients who were discharged from the surgical hospitalization alive and therefore at risk for unplanned readmission.

\section{Missing Data}

Patients with missing demographic data were placed in a separate category for that variable, allowing for appropriate use of missing data in logistic regression. Data on the diagnosis associated with readmission were available from 2012 to 2014 but missing for some patients within this time period; a sensitivity analysis compared major complications among patients with a recorded and missing readmission diagnosis, to evaluate if any selection bias was introduced by the missing data.

\section{Statistical Analysis}

Statistical analyses were conducted in SPSS (version 23, IBM Corp.). Descriptive statistics were performed on baseline variables, and univariable logistic regression assessed their association with the specific outcome evaluated. Multivariable logistic regression models were thereafter constructed minimizing statistical optimism, maximizing parsimony, and avoiding overfitting by following the rule of a maximum of 1 covariate for 10 events. Variables were screened with univariable logistic regression to evaluate for differences within any strata of categorical variables, and those with a $p$ value $<0.10$ were evaluated for inclusion in the final model, which was determined using backward selection with the likelihood ratio test. Concordance statistics determined the discrimination of logistic regression constructs, which range between 0.50 , where a model has no greater discrimination than chance alone, to 1.0, where the model has perfect discrimination. Calibration of models was assessed using the HosmerLemeshow test, which tests the null hypothesis that the observed and expected counts in each decile are equal; a model is accepted when the probability value indicates failure to reject the null hypothesis. A probability value < 0.05 was determined to be statistically significant.

\section{Results}

\section{Demographics of the Study Population}

Within the study population, 2207 patients were included and $51.4 \%$ of tumors were extradural, $36.4 \%$ were intradural extramedullary, and $12.3 \%$ were intramedullary. Tumors by location were $20.7 \%$ cervical, $47.4 \%$ thoracic, $29.1 \%$ lumbar, and $2.8 \%$ sacral. The baseline characteristics and treatment variables are compared by unplanned readmission in Table 1.

\section{Cumulative Incidence and Time to Event of Complications}

The 30-day cumulative incidence and time to event of complications (that are recorded by the NSQIP algorithm) are listed in Table 2. Major complications occurred in $14.4 \%$ of patients. The most common complications and their median times to occurrence were VTE (4.5\%) at 9 days (interquartile range [IQR] 4-19 days) postoperatively, SSIs (3.6\%) at 18 days (IQR 14-25 days), sepsis (2.9\%) at 13 days (IQR 7-21 days), and pneumonia (2.7\%) at 9 days (IQR 3-15 days). The median hospital length of stay 
TABLE 1. Demographics of the study population, stratified by 30 -day readmission*

\begin{tabular}{|c|c|c|c|c|c|c|}
\hline \multirow[b]{2}{*}{ Definition } & \multicolumn{6}{|c|}{ Unplanned Readmissions } \\
\hline & Total & Yes & No & OR & $95 \% \mathrm{Cl}$ & $\mathrm{p}$ Value \\
\hline \multicolumn{7}{|l|}{ Spinal level } \\
\hline Cervical & 20.7 & 18.9 & 20.9 & Ref & & \\
\hline Thoracic & 47.4 & 55.0 & 46.5 & 1.31 & $0.90-1.89$ & 0.16 \\
\hline Lumbar & 29.1 & 23.9 & 29.7 & 0.89 & $0.58-1.36$ & 0.60 \\
\hline Sacral & 2.80 & 2.30 & 2.90 & 0.86 & $0.33-2.27$ & 0.77 \\
\hline \multicolumn{7}{|l|}{ Location } \\
\hline Extradural & 51.4 & 62.2 & 50.1 & Ref & & \\
\hline Intradural extramedullary & 36.4 & 24.8 & 37.7 & 0.53 & $0.38-0.74$ & $<0.001$ \\
\hline Intramedullary & 12.3 & 13.1 & 12.2 & 0.87 & $0.57-1.32$ & 0.51 \\
\hline \multicolumn{7}{|l|}{ Sex } \\
\hline Female & 45.5 & 41.4 & 46.0 & Ref & & \\
\hline Male & 54.5 & 58.6 & 54.0 & 1.20 & $0.91-1.60$ & 0.20 \\
\hline \multicolumn{7}{|l|}{ Race/ethnicity } \\
\hline Caucasian & 70.8 & 74.8 & 70.4 & Ref & & \\
\hline African-American & 8.30 & 9.90 & 8.10 & 1.15 & $0.71-1.84$ & 0.58 \\
\hline Asian & 4.80 & 3.60 & 4.90 & 0.69 & $0.33-1.45$ & 0.33 \\
\hline Hispanic & 6.70 & 7.20 & 6.70 & 1.02 & $0.59-1.76$ & 0.95 \\
\hline Not reported & 9.30 & 4.50 & 9.90 & 0.43 & $0.22-0.83$ & 0.01 \\
\hline \multicolumn{7}{|l|}{ Age quartile (yrs) } \\
\hline Lower (<46) & 25.2 & 19.0 & 25.9 & Ref & & \\
\hline Second (46-58) & 24.8 & 23.1 & 25.1 & 1.25 & $0.82-1.92$ & 0.30 \\
\hline Third (58-67) & 25.1 & 26.2 & 24.9 & 1.43 & $0.95-2.17$ & 0.09 \\
\hline Upper (>67) & 24.9 & 31.7 & 24.1 & 1.79 & $1.20-2.68$ & 0.005 \\
\hline \multicolumn{7}{|l|}{ BMI } \\
\hline Non-obese & 62.7 & 62.2 & 62.8 & Ref & & \\
\hline Class I (30-35 kg/m²) & 19.0 & 19.8 & 18.9 & 1.06 & $0.74-1.52$ & 0.75 \\
\hline Class II/III $\left(\geq 35 \mathrm{~kg} / \mathrm{m}^{2}\right)$ & 15.5 & 14.4 & 15.7 & 0.93 & $0.62-1.39$ & 0.72 \\
\hline Missing & 2.70 & 3.60 & 2.60 & 1.38 & $0.64-2.98$ & 0.41 \\
\hline \multicolumn{7}{|l|}{ Functional health status } \\
\hline Independent & 91.1 & 86.5 & 91.6 & Ref & & \\
\hline Dependent & 8.50 & 12.6 & 8.00 & 1.67 & $1.09-2.57$ & 0.02 \\
\hline Missing & 0.40 & 0.90 & 0.40 & 2.64 & $0.55-12.8$ & 0.28 \\
\hline \multicolumn{7}{|l|}{ Diabetes mellitus } \\
\hline No & 88.2 & 86.9 & 88.3 & Ref & & \\
\hline Insulin & 4.80 & 7.20 & 4.50 & 1.61 & $0.93-2.80$ & 0.09 \\
\hline Non-insulin & 7.00 & 5.90 & 7.20 & 0.83 & $0.46-1.49$ & 0.53 \\
\hline Current smoker & 17.2 & 21.2 & 16.8 & 1.33 & $0.95-1.88$ & 0.10 \\
\hline Dyspnea & 5.80 & 13.5 & 50.0 & 3.00 & $1.94-4.64$ & $<0.001$ \\
\hline COPD & 3.20 & 5.40 & 2.90 & 1.88 & $0.99-3.57$ & 0.05 \\
\hline Hypertension & 42.8 & 50.9 & 41.9 & 1.44 & $1.09-1.90$ & 0.01 \\
\hline Disseminated cancer & 29.8 & 46.4 & 27.8 & 2.24 & $1.69-2.97$ & $<0.001$ \\
\hline \multicolumn{7}{|c|}{ Recent chemotherapy or radiotherapy } \\
\hline No & 11.9 & 8.60 & 12.3 & Ref & & \\
\hline Yes & 1.20 & 2.30 & 1.10 & 3.00 & $1.02-8.83$ & 0.047 \\
\hline Missing & 86.9 & 89.2 & 86.6 & 1.48 & $0.91-2.42$ & 0.91 \\
\hline Open wound & 1.90 & 2.70 & 1.80 & 1.51 & $0.63-3.63$ & 0.36 \\
\hline Steroid use & 9.70 & 18.0 & 8.80 & 2.29 & $1.57-3.33$ & $<0.001$ \\
\hline Weight loss & 3.60 & 5.00 & 3.50 & 1.46 & $0.76-2.80$ & 0.26 \\
\hline
\end{tabular}


» CONTINUED FROM PAGE 4

TABLE 1. Demographics of the study population, stratified by 30 -day readmission*

\begin{tabular}{|c|c|c|c|c|c|c|}
\hline \multirow[b]{2}{*}{ Definition } & \multicolumn{6}{|c|}{ Unplanned Readmissions } \\
\hline & Total & Yes & No & OR & $95 \% \mathrm{Cl}$ & $\mathrm{p}$ Value \\
\hline Bleeding disorders & 3.40 & 7.20 & 3.00 & 2.52 & $1.42-4.46$ & 0.002 \\
\hline Preop transfusion & 1.90 & 5.40 & 1.40 & 3.90 & $1.95-7.78$ & $<0.001$ \\
\hline Preop SIRS & 4.20 & 7.20 & 3.90 & 1.93 & $1.10-3.38$ & 0.02 \\
\hline \multicolumn{7}{|l|}{ Wound class } \\
\hline Class I & 97.9 & 97.3 & 98.0 & Ref & & \\
\hline Class II-IV & 2.10 & 2.70 & 2.00 & 1.35 & $0.57-3.23$ & 0.50 \\
\hline \multicolumn{7}{|l|}{ ASA Class } \\
\hline $1-2$ & 36.2 & 20.7 & 38 & Ref & & \\
\hline 3 & 53.7 & 62.2 & 52.7 & 2.16 & $1.53-3.06$ & $<0.001$ \\
\hline $4-5$ & 10.0 & 17.1 & 9.20 & 3.40 & $2.15-5.38$ & $<0.001$ \\
\hline Emergency case & 9.50 & 14.4 & 9.00 & 1.71 & $1.14-2.56$ & 0.01 \\
\hline \multicolumn{7}{|l|}{ Total op time quartile (min) } \\
\hline Lower (<136) & 24.2 & 23.9 & 24.3 & Ref & & \\
\hline Second (136-196) & 25.5 & 23.9 & 25.7 & 0.95 & $0.63-1.41$ & 0.79 \\
\hline Third (196-272) & 25.1 & 22.1 & 25.4 & 0.88 & $0.59-1.33$ & 0.55 \\
\hline Upper (>272) & 25.2 & 30.2 & 24.6 & 1.25 & $0.85-1.83$ & 0.26 \\
\hline \multicolumn{7}{|l|}{ Preop labs } \\
\hline \multicolumn{7}{|l|}{ Creatinine (mg/dl) } \\
\hline$<1.4$ & 89.4 & 92.3 & 89.0 & Ref & & \\
\hline$>1.4$ & 4.80 & 5.90 & 4.7 & 1.20 & $0.66-2.19$ & 0.55 \\
\hline Not obtained & 5.80 & 1.80 & 6.30 & 0.28 & $0.10-0.76$ & 0.01 \\
\hline \multicolumn{7}{|l|}{ Sodium (mEq/L) } \\
\hline$>135$ & 78.0 & 75.7 & 78.2 & Ref & & \\
\hline$<135$ & 15.5 & 22.5 & 14.7 & 1.58 & $1.12-2.22$ & 0.009 \\
\hline Not obtained & 6.50 & 1.80 & 7.00 & 0.27 & $0.10-0.72$ & 0.01 \\
\hline \multicolumn{7}{|l|}{ Albumin (g/dl) } \\
\hline$>3.5$ & 36.0 & 40.1 & 35.6 & Ref & & \\
\hline$<3.5$ & 16.1 & 29.7 & 14.5 & 1.81 & $1.28-2.57$ & 0.001 \\
\hline Not obtained & 47.8 & 30.2 & 49.9 & 0.54 & $0.39-0.75$ & $<0.001$ \\
\hline \multicolumn{7}{|l|}{ 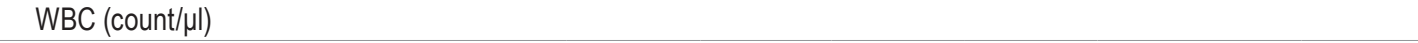 } \\
\hline $4-12,000$ & 79.2 & 75.2 & 79.7 & Ref & & \\
\hline$<4000$ & 4.00 & 5.90 & 3.80 & 1.65 & $0.89-3.04$ & 0.11 \\
\hline$>12,000$ & 12.7 & 17.6 & 12.1 & 1.54 & $1.06-2.23$ & 0.025 \\
\hline Not obtained & 4.10 & 1.40 & 4.40 & 0.32 & $0.10-1.03$ & 0.06 \\
\hline \multicolumn{7}{|l|}{ Hematocrit (\%) } \\
\hline$<36$ & 71.9 & 61.7 & 73.1 & Ref & & \\
\hline$>36$ & 24.4 & 36.9 & 22.9 & 1.91 & $1.42-2.56$ & $<0.001$ \\
\hline Not obtained & 3.70 & 1.40 & 4.00 & 0.40 & $0.13-1.29$ & 0.13 \\
\hline \multicolumn{7}{|l|}{ PTT (sec) } \\
\hline$<40$ & 69.4 & 72.5 & 69.1 & Ref & & \\
\hline$>40$ & 1.80 & 1.80 & 1.80 & 0.98 & $0.34-2.80$ & 0.97 \\
\hline Not obtained & 28.8 & 25.7 & 29.2 & 0.84 & $0.61-1.15$ & 0.27 \\
\hline \multicolumn{7}{|l|}{ INR } \\
\hline$<1.4$ & 81.4 & 84.7 & 81.0 & Ref & & \\
\hline$>1.4$ & 1.40 & 2.70 & 1.20 & 2.10 & $0.84-5.18$ & 0.11 \\
\hline Not obtained & 17.2 & 12.6 & 17.7 & 0.68 & $0.45-1.03$ & 0.07 \\
\hline
\end{tabular}


» CONTINUED FROM PAGE 5

TABLE 1. Demographics of the study population, stratified by 30 -day readmission*

\begin{tabular}{|c|c|c|c|c|c|c|}
\hline \multirow[b]{2}{*}{ Definition } & \multicolumn{6}{|c|}{ Unplanned Readmissions } \\
\hline & Total & Yes & No & OR & $95 \% \mathrm{Cl}$ & p Value \\
\hline \multicolumn{7}{|l|}{ Preop labs (continued) } \\
\hline \multicolumn{7}{|l|}{ LOS quartile (days) } \\
\hline Lower $(<3)$ & 28.7 & 14.0 & 30.4 & Ref & & \\
\hline Second (3-5) & 22.5 & 20.3 & 22.7 & 1.94 & $1.21-3.12$ & 0.006 \\
\hline Third (5-9) & 25.9 & 32.9 & 25.1 & 2.86 & $1.84-4.42$ & $<0.001$ \\
\hline Upper (>9) & 23.0 & 32.9 & 21.8 & 3.27 & $2.11-5.07$ & $<0.001$ \\
\hline \multicolumn{7}{|c|}{ Complications during surgical hospitalization } \\
\hline Cardiopulmonary & 1.40 & 0.90 & 1.50 & 0.60 & $0.14-2.53$ & 0.49 \\
\hline VTE & 2.10 & 3.60 & 1.90 & 1.92 & $0.88-4.18$ & 0.10 \\
\hline Infectious & 4.40 & 4.10 & 4.40 & 0.91 & $0.45-1.84$ & 0.79 \\
\hline Transfusion & 19.5 & 26.1 & 18.7 & 1.54 & $1.11-2.11$ & 0.009 \\
\hline \multicolumn{7}{|l|}{ Discharge destination } \\
\hline Home & 64.9 & 53.6 & 66.2 & Ref & & \\
\hline Nonroutine & 34.6 & 46.4 & 33.2 & 1.73 & $1.30-2.28$ & $<0.001$ \\
\hline Missing & 0.50 & 0.00 & 0.60 & - & - & - \\
\hline
\end{tabular}

(LOS) was 5 days (IQR 3-9 days). The 30-day mortality rate was $3.3 \%$, with the median time to death being 20 days (IQR 12.5-26 days) postoperatively. The discharge disposition of patients was home for $64.9 \%$ of patients and nonroutine (rehabilitation or an acute care facility) for the remaining $34.6 \%$.

A multivariable logistic regression model was constructed to evaluate the predictors of a major complication, defined as death, postoperative stroke or coma, cardiopulmonary complications, symptomatic venous thrombosis stroke, acute renal failure, sepsis, or an SSI (Table 3). The independent predictors of a major complication were (by descending effect size): ASA Class 4-5 designation, bleeding disorders, ASA Class 3 designation, preoperative SIRS, preoperative leukocytosis ( $>12,000 / \mu \mathrm{l})$, emergency surgery, dependent functional health status, longer opera-

TABLE 2. Cumulative incidence and time to event of postoperative complications

\begin{tabular}{|c|c|c|c|}
\hline \multirow[b]{2}{*}{ Variable } & \multirow{2}{*}{$\begin{array}{c}\text { Cumulative } \\
\text { Incidence (\%) }\end{array}$} & \multicolumn{2}{|c|}{ Time to Event (days) } \\
\hline & & Median & IQR \\
\hline Cardiac complication & 0.5 & 8.0 & $3.5-16.5$ \\
\hline CNS complication & 0.2 & 18.5 & $3.8-23.8$ \\
\hline Pneumonia & 2.7 & 9.0 & $3.0-15.0$ \\
\hline Pulmonary complication & 2.2 & 5.0 & $2.0-13.0$ \\
\hline Renal complication & 0.3 & 13.0 & $9.0-15.0$ \\
\hline Sepsis & 2.9 & 13.0 & $7.0-21.0$ \\
\hline SSI & 3.6 & 18.0 & $14.0-25.0$ \\
\hline Transfusions & 19.7 & 0.0 & $0.0-0.0$ \\
\hline VTE & 4.5 & 9.0 & $4.0-19.0$ \\
\hline UTI & 3.7 & 11.0 & $6.0-17.8$ \\
\hline
\end{tabular}

tive time (upper quartile $>272$ minutes), preoperative dyspnea, and male sex $(\mathrm{p}<0.05)$; preoperative steroid use was predictive of a major complication (of borderline significance). The model had good discrimination (area under the receiver operating curve $[\mathrm{AUROC}]=0.73$ ), and appropriate calibration (Hosmer-Lemeshow test $=0.39$ ).

\section{Readmission}

Unplanned readmission occurred in $10.2 \%(\mathrm{n}=222)$ of patients discharged from the surgical hospital alive, and occurred at a median of 18 days (IQR 12-23 days) postoperatively and 10 days (IQR 5-15 days) after discharge. Multivariable logistic regression models were constructed and the independent predictors of readmission, in descending effect size, were preoperative transfusions, LOS $>3$ days, preoperative dyspnea, preoperative steroid administration, disseminated cancer, and hypertension (Table 4). The model was found to have moderate discrimination $($ AUROC $=0.678)$ and good calibration (Hosmer-Lemeshow test $=0.67$ ).

Of patients who were readmitted between 2012 and 2014, 65.9\% $(\mathrm{n}=118)$ had a documented diagnosis associated with readmission. The most common reasons for unplanned readmission were SSIs (23.7\%), systemic infections (17.8\%), VTE (12.7\%), CNS complications (11.9\%), gastrointestinal (GI)/genitourinary (GU) complications (7.6\%), pain (5.1\%), fever (4.2\%), pulmonary or cardiac complications (4.1\%), and other (12.7\%). Among SSIs requiring readmission, superficial infections were the most common (42.9\%) while the remaining (57.1\%) were deep (Fig. 1 left). Of the patients with systemic infections that required readmission, $38.0 \%$ were for sepsis, $28.5 \%$ were for pneumonia, and the remaining for other systemic infections including UTIs and meningitis. Kaplan-Meier 
TABLE 3. Multivariable logistic regression evaluating predictors of a major complication*

\begin{tabular}{|c|c|c|c|}
\hline \multirow[b]{2}{*}{ Variable } & \multicolumn{3}{|c|}{ Serious Adverse Events } \\
\hline & OR & $95 \% \mathrm{Cl}$ & p Value \\
\hline \multicolumn{4}{|l|}{ Sex } \\
\hline Female & Ref & & \\
\hline Male & 1.33 & $1.02-1.73$ & 0.03 \\
\hline \multicolumn{4}{|l|}{ Functional health status } \\
\hline Independent & Ref & & \\
\hline Dependent & 1.72 & $1.18-2.51$ & 0.005 \\
\hline Missing & 2.05 & $0.40-10.5$ & 0.39 \\
\hline Current smoker & 1.33 & $0.96-1.82$ & 0.08 \\
\hline Dyspnea & 1.62 & $1.04-2.52$ & 0.03 \\
\hline Steroid use & 1.43 & $0.99-2.07$ & 0.05 \\
\hline Bleeding disorders & 2.87 & $1.74-4.74$ & $<0.001$ \\
\hline Preop transfusion & 1.86 & $0.95-3.66$ & 0.07 \\
\hline Preop SIRS & 1.85 & $1.14-3.01$ & 0.01 \\
\hline \multicolumn{4}{|l|}{ Wound class } \\
\hline Class I & Ref & & \\
\hline Class II-IV & 1.97 & $0.96-4.03$ & 0.06 \\
\hline \multicolumn{4}{|l|}{ ASA Class } \\
\hline $1-2$ & Ref & & \\
\hline 3 & 2.01 & $1.44-2.80$ & $<0.001$ \\
\hline $4-5$ & 3.07 & $1.97-4.80$ & $<0.001$ \\
\hline Emergency case & 1.73 & $1.20-2.50$ & 0.003 \\
\hline \multicolumn{4}{|c|}{ Total op time quartile (min) } \\
\hline Lower $(<136)$ & Ref & & \\
\hline Second (136-196) & 0.98 & $0.68-1.43$ & 0.93 \\
\hline Third (196-272) & 1.03 & $0.70-1.50$ & 0.90 \\
\hline Upper (>272) & 1.63 & $1.14-2.32$ & 0.007 \\
\hline \multicolumn{4}{|l|}{ WBC (count/pl) } \\
\hline $4000-12,000$ & Ref & & \\
\hline$<4000$ & 1.21 & $0.68-2.17$ & 0.52 \\
\hline$>12,000$ & 1.82 & $1.30-2.53$ & $<0.001$ \\
\hline Not obtained & 1.17 & $0.57-2.43$ & 0.67 \\
\hline
\end{tabular}

* Boldface type indicates statistical significance. Hosmer-Lemeshow test $=$ 0.39, AUROC $=0.728$.

curves depicting time to event analysis of unplanned readmissions, as well as SSI, VTE, and unplanned reoperations, are depicted in Fig. 2.

Because the ICD-9-CM code associated with readmission was not recorded in 2011 or available in all patients from 2012 to 2014, a sensitivity analysis was performed of patients who were readmitted, evaluating if those with a known diagnosis experienced a differential complication rate compared with those without a recorded diagnosis. Recorded readmission diagnosis was not significantly associated with the development of a major complication, suggesting that missing data on readmission diagnosis did not introduce a selection bias (data not shown).

\section{Reoperation}

Unplanned reoperation occurred in 5.3\% ( $\mathrm{n}=95)$ of pa- tients at a median of 13 days (IQR 8-20 days) postoperatively. Independent predictors of reoperation were preoperative steroid use and ASA classification 4-5. The CPT code indicating the procedure that was performed at the time of reoperation was documented in $97.9 \%(n=93)$ of patients. The most common reason for unplanned reoperation was SSI (32\%, Fig. 1 right). Additional reasons for reoperation were spinal reoperation (26\%), extraspinal operation (25\%), CSF leak repair (13\%), and CNS-related reoperation (4\%).

\section{Discussion}

The NSQIP database has been increasingly used to examine patient outcomes following spine surgery, including readmission after reconstruction of spinal deformity and after spinal fusion. ${ }^{59,91}$ Nevertheless, few studies have used the NSQIP database to evaluate the outcome of patients undergoing surgery for spinal tumors. Schoenfeld et al. used the NSQIP database to validate the ability of the New England Spinal Metastasis Score to predict adverse outcomes including mortality, complications, and failure to rescue in patients with secondary spinal tumors; the score is based on extent of malignant spread, preoperative albumin, and ambulatory capacity. ${ }^{77}$ Nevertheless, this study did not build predictive models of adverse outcomes or evaluate the reasons for reoperation or readmission, and it was restricted to patients with metastatic lesions. There is a relative dearth of literature evaluating the incidence and predictors of adverse events after surgery for spinal tumors, and prior publications have been primarily based on retrospective, institutional studies. The goal of this analysis was to specifically evaluate the 30day cumulative incidence, time to event, and predictors of readmission, reoperation, and postoperative complications for patients undergoing surgery for primary and secondary spinal tumors.

In our analysis, the 30-day readmission rate was $10.2 \%$; the most common reasons for readmission were SSIs (23.7\%), systemic infections (17.8\%), VTE (12.7\%), and CNS complications (11.9\%). A recent meta-analysis of 13 retrospective studies reported a pooled 30-day readmission rate of $6.1 \%$ (95\% confidence interval [CI] $4.1 \%-8.9 \%$ ) in studies with patients undergoing surgery for all spinal levels for elective or emergency procedures. ${ }^{10}$ Another national database study of 343,068 patients over the age of 65 with public insurance (Medicare) who underwent elective spinal surgery for degenerative conditions demonstrated a 30 -day readmission rate of $7.9 \%$ for patients undergoing cervical spine surgery and $7.3 \%$ for those undergoing lumbar spine surgery. ${ }^{94}$ In this study, there was a higher 30-day readmission rate compared with studies evaluating other spinal pathologies, highlighting that oncology patients suffer a greater burden of systemic illness than patients with other spinal pathologies, thereby resulting in their increased readmission rates. Allaudeen et al. showed in multivariable analysis of more than 10,000 consecutive general medicine patients in a tertiary care academic medical center that cancer with or without metastasis was a factor associated with readmission. ${ }^{5} \mathrm{~A}$ retrospective single-institution cohort study by Schairer et al. similarly found a higher rate of readmission for patients with primary and metastatic tumors of the spine. In their study, they reported a readmission rate of $14.2 \%{ }^{74}$ 
TABLE 4. Multivariable logistic regression models evaluating predictors of unplanned readmission and unplanned reoperation*

\begin{tabular}{|c|c|c|c|c|c|c|}
\hline \multirow[b]{2}{*}{ Variable } & \multicolumn{3}{|c|}{ Unplanned Readmission† } & \multicolumn{3}{|c|}{ Unplanned Reoperation $\ddagger$} \\
\hline & OR & $95 \% \mathrm{Cl}$ & $p$ Value & OR & $95 \% \mathrm{Cl}$ & $\mathrm{p}$ Value \\
\hline \multicolumn{7}{|l|}{ Location } \\
\hline Extradural & - & - & - & Ref & & \\
\hline Intradural extramedullary & - & - & - & 0.60 & $0.35-1.02$ & 0.06 \\
\hline Intramedullary & - & - & - & 1.24 & $0.67-2.31$ & 0.50 \\
\hline Dyspnea & 2.17 & $1.37-3.43$ & 0.001 & - & - & - \\
\hline Hypertension & 1.38 & $1.04-1.84$ & 0.03 & - & - & - \\
\hline Disseminated cancer & 1.53 & $1.13-2.09$ & 0.007 & - & - & - \\
\hline Steroid use & 1.77 & $1.19-2.62$ & 0.005 & 2.06 & $1.20-3.54$ & 0.009 \\
\hline Preop transfusion & 2.60 & $1.26-5.40$ & 0.01 & - & - & - \\
\hline Preop SIRS & - & - & - & 1.94 & $0.93-4.03$ & 0.08 \\
\hline \multicolumn{7}{|l|}{ ASA Class } \\
\hline $1-2$ & - & - & - & Ref & & \\
\hline 3 & - & - & - & 1.60 & $0.94-2.73$ & 0.09 \\
\hline $4-5$ & - & - & - & 2.58 & $1.29-5.18$ & 0.007 \\
\hline \multicolumn{7}{|l|}{ LOS quartile (days) } \\
\hline Lower $(<3)$ & Ref. & & & - & - & - \\
\hline Second (3-5) & 1.80 & $1.11-2.91$ & 0.02 & - & - & - \\
\hline Third (5-9) & 2.32 & $1.48-3.65$ & $<0.001$ & - & - & - \\
\hline Upper (>9) & 2.36 & $1.48-3.74$ & $<0.001$ & - & - & - \\
\hline
\end{tabular}

and also confirmed that infectious complications were the most common cause for readmission. ${ }^{10}$ Similar to previous prognostic single- and multiinstitutional studies, on multivariate analysis we identified comorbidities (dyspnea, hypertension, anemia), disseminated cancer, preoperative steroid use, and an extended hospitalization as predictors of readmission after spine tumor surgery. In contrast to the meta-analysis by Bernatz and Anderson of factors predicting readmission in any type of spine surgery, ASA classifi- cation of 4+ was not a significant predictor in this NSQIP database sample of spinal tumors studied..$^{10}$ In addition, limitations of the NSQIP database prevented the ability to assess the predictive value of demographical factors such as preoperative insurance status on postoperative outcomes.

In spine surgery, rates of reoperation have varied by surgical approach, extent of the procedure, and patient population demographics. ${ }^{43,55,73,75}$ For example, Kukreja
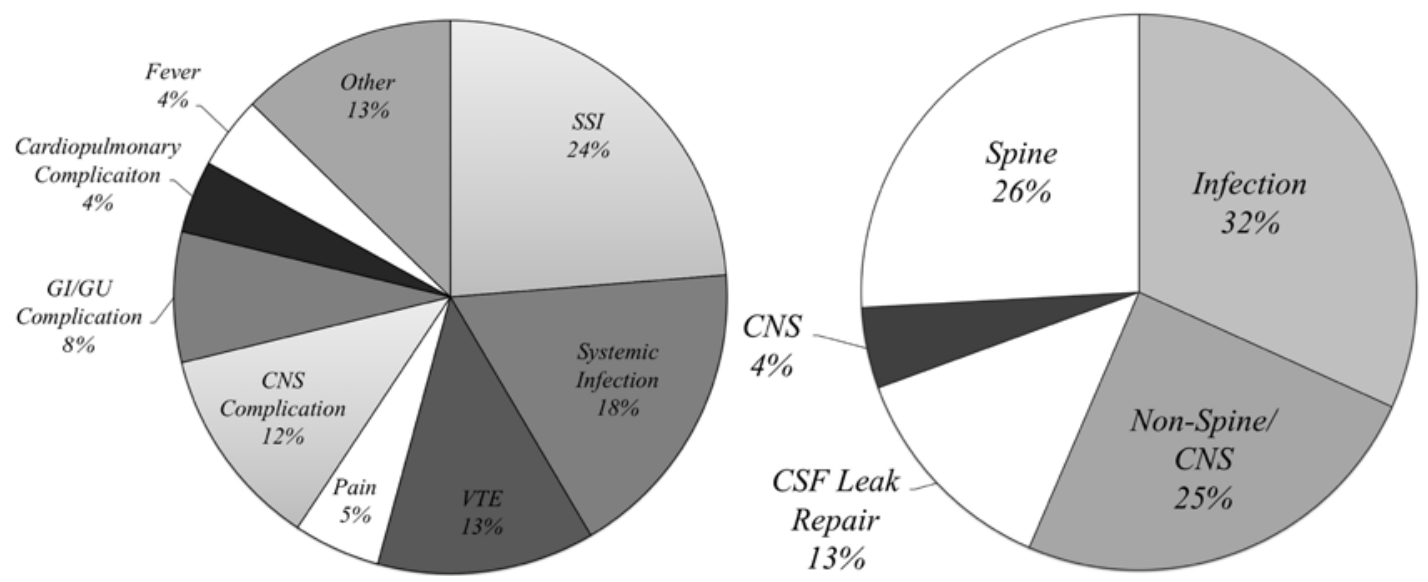

FIG. 1. Pie chart depicting the reasons for unplanned readmissions (left) and unplanned reoperations (right). The primary reason for readmission was available for $65.9 \%$ of readmitted patients from 2012 to 2014 . The primary reason for reoperation was available for $97.9 \%$ of reoperated patients from 2012 to 2014 . 

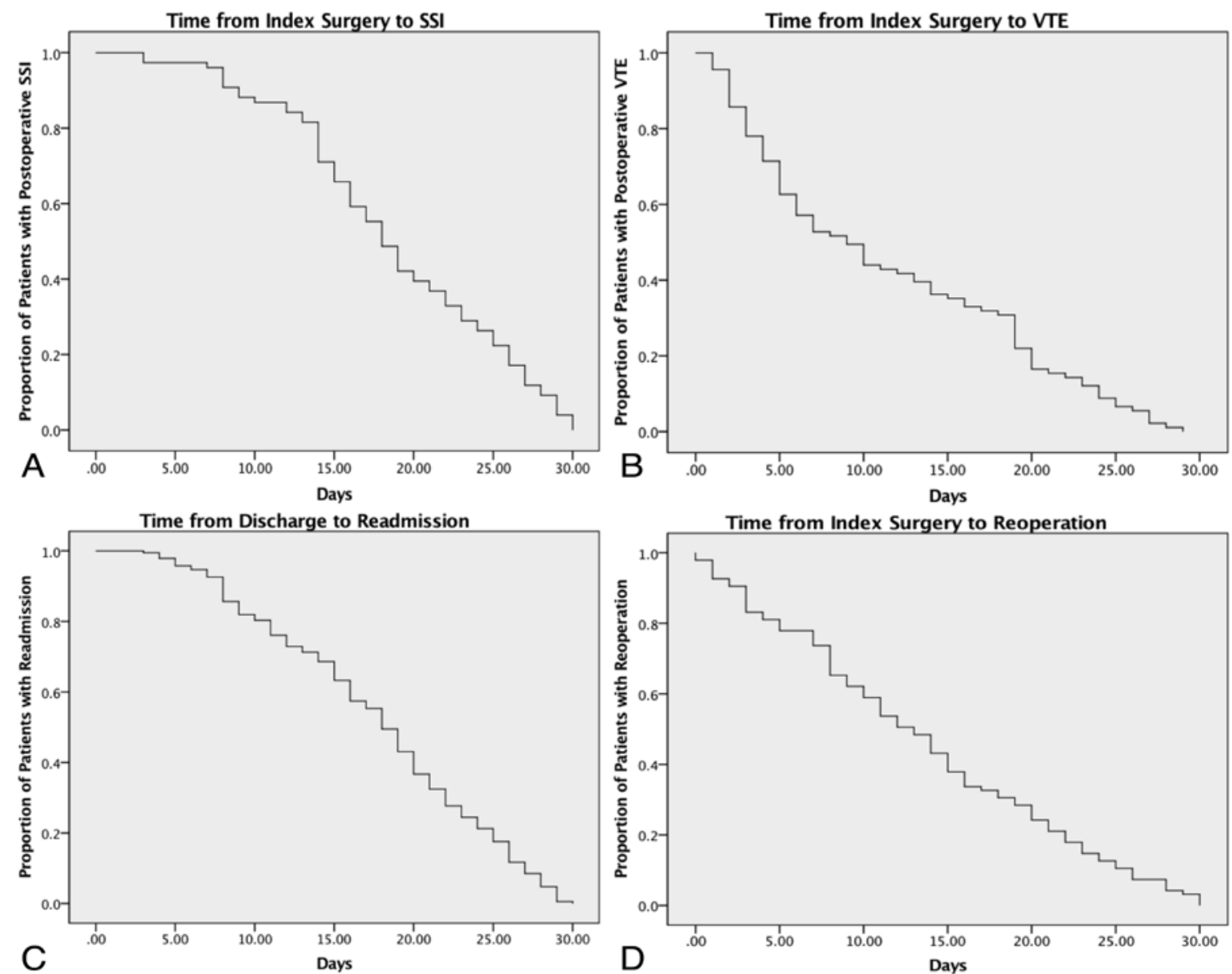

FIG. 2. Kaplan-Meier curves depicting time to event analysis of SSI (A), VTE (B), unplanned readmissions (C), and unplanned reoperations (D).

et al. found in a single-institution retrospective study that $41.3 \%$ of patients who underwent decompressive lumbar spine surgeries for on-the-job injuries required reoperation, usually within 2 years. ${ }^{55}$ A different retrospective study reported a $6.1 \%$ reoperation rate at 1 year for patients who underwent surgery for degenerative spondylolisthesis. ${ }^{73}$ Scheer et al. performed a retrospective review of an adult spinal deformity database and found that $17 \%$ of patients underwent any reoperation, and 5\% underwent reoperation within 30 days. ${ }^{75}$ In our study, we found that reoperation occurred in 5.3\% of patients with primary and secondary spinal tumors at a median of 13 days postoperatively. A recent single-institution ambispective review of 289 patients who underwent surgery for spinal metastases revealed a $10.7 \%$ reoperation rate, the majority of which took place during the same admission. ${ }^{72}$ In our study, reoperation was also found to be associated with preoperative steroid use and ASA classification 4-5.

Surgery for spinal tumors is known to be associated with a higher rate of complications compared with degenerative procedures, partially due to the burden of disease of cancer patients, particularly those with metastases. In a prospective study of patients undergoing emergency oncological spinal surgery, Dea et al. reported that $76.2 \%$ of patients had 1 or more adverse events and $10.9 \%$ died during admis- sion..$^{32}$ In our study, the most common complications were VTE (4.5\%), SSIs (3.6\%), and sepsis (2.9\%). Predictors of an adverse event were dependent functional status, preoperative steroid use, leukocytosis, SIRS, ASA Class 3-5 designation, emergency operation, and longer operative time.

While the predictors of adverse events, reoperations, and readmissions are noteworthy, equally interesting were variables screened but not found to be associated with adverse events. First, neither tumor location (extradural, intradural extramedullary, or intramedullary) nor spinal location was predictive of complications, reoperations, or readmissions. Moreover, race/ethnicity was not associated with differential outcomes, despite the fact that disparities are well-known in oncology patients. ${ }^{31}$ While obesity has been shown to be associated with adverse events among patients undergoing surgery for degenerative spinal conditions ${ }^{89}$ in this analysis body habitus was not associated with differential outcomes. Additionally, neither complications during the surgical hospitalization nor discharge disposition were predictive of readmission.

The data on complications and readmission highlight the frequency of SSIs, VTE, and systemic infections in patients undergoing surgery for spinal tumors. The chronological association of these complications showed that they occurred late, often after discharge from the surgical hos- 
pital stay. Additionally, complications during the surgical hospital stay were not predictive of readmission, emphasizing that patients are readmitted for new postdischarge complications, rather than for exacerbations of postoperative complications that occurred during the surgical hospitalization. Therefore, patients undergoing resection of spinal tumors require particular surveillance for these infectious and venous thromboembolic complications after discharge. Moreover, postdischarge VTE prophylaxis has been used in oncology patients undergoing other surgical procedures, and future studies may be merited to evaluate if there is utility to similar practice among patients undergoing spinal tumor surgery. ${ }^{64,76}$

However, there are many limitations to using the NSQIP database to evaluate outcomes following spine surgery. Data are not collected on the neurological or functional outcomes after surgery and intraoperative complications are not tracked. Additionally, tumor size and preoperative neurological function could not be assessed, and data on preoperative chemotherapy or radiation were missing for many patients (as they are not collected in the most recent NSQIP database algorithms). Therefore, the NSQIP database lacks a certain degree of clinical granularity to discern outcomes of neurosurgical patients. Moreover, the NSQIP database only tracks patients for 30 days postoperatively, and therefore long-term outcomes including mortality, neurological function, and pseudarthrosis could not be evaluated.

Nevertheless, there are many distinct advantages to using the NSQIP database to evaluate the outcomes of patients with spinal tumors. The large patient population accrued by the NSQIP database provides the statistical power to discern many predictors of complications. Additionally, the national perspective increases the generalizability of results beyond single-center reports. Future studies will be needed to evaluate transitional care programs that may reduce the readmission rate of patients undergoing spine surgery.

\section{Conclusions}

In this national multiinstitution analysis of patients undergoing spine surgery for primary and secondary spinal tumors, $10.2 \%$ were readmitted within 30 days after discharge. The most common reasons for readmission were SSIs, systemic infections, and VTE, which were late complications that often occurred after discharge from the surgical hospitalization. Additionally, patients were primarily readmitted for new complications that developed following discharge rather than exacerbation of complications from the initial surgical hospital stay. Overall, 5.3\% underwent reoperation, $14.4 \%$ experienced a major complication, and 3.3\% died within 30 days postoperatively. This analysis provides predictive models for surgeons to identify and risk-stratify patients who may benefit from increased surveillance following discharge, including transitional care programs.

\section{References}

1. Adams H, Avendaño J, Raza SM, Gokaslan ZL, Jallo GI, Quiñones-Hinojosa A: Prognostic factors and survival in primary malignant astrocytomas of the spinal cord: a pop- ulation-based analysis from 1973 to 2007. Spine (Phila Pa 1976) 37:E727-E735, 2012

2. Akins PT, Harris J, Alvarez JL, Chen Y, Paxton EW, Bernbeck J, et al: Risk factors associated with 30-day readmissions after instrumented spine surgery in 14,939 patients: 30-day readmissions after instrumented spine surgery. Spine (Phila Pa 1976) 40:1022-1032, 2015

3. Aldebeyan S, Aoude A, Fortin M, Nooh A, Eng PJ, Ouellet $\mathrm{J}$, et al: Predictors of discharge destination after lumbar spine fusion surgery. Spine (Phila Pa 1976) [epub ahead of print], 2016

4. Algattas H, Kimmell KT, Vates GE: Risk of reoperation for hemorrhage in patients after craniotomy. World Neurosurg 87:531-539, 2016

5. Allaudeen N, Vidyarthi A, Maselli J, Auerbach A: Redefining readmission risk factors for general medicine patients. J Hosp Med 6:54-60, 2011

6. Basques BA, Chung SH, Lukasiewicz AM, Webb ML, Samuel AM, Bohl DD, et al: Predicting short-term morbidity in patients undergoing posterior spinal fusion for neuromuscular scoliosis. Spine (Phila Pa 1976) 40:1910-1917, 2015

7. Basques BA, Fu MC, Buerba RA, Bohl DD, Golinvaux NS, Grauer JN: Using the ACS-NSQIP to identify factors affecting hospital length of stay after elective posterior lumbar fusion. Spine (Phila Pa 1976) 39:497-502, 2014

8. Bekelis K, Bakhoum SF, Desai A, Mackenzie TA, Roberts DW: Outcome prediction in intracranial tumor surgery: the National Surgical Quality Improvement Program 20052010. J Neurooncol 113:57-64, 2013

9. Bekelis K, Desai A, Bakhoum SF, Missios S: A predictive model of complications after spine surgery: the National Surgical Quality Improvement Program (NSQIP) 20052010. Spine J 14:1247-1255, 2014

10. Bernatz JT, Anderson PA: Thirty-day readmission rates in spine surgery: systematic review and meta-analysis. Neurosurg Focus 39(4):E7, 2015

11. Bilsky MH, Laufer I, Fourney DR, Groff M, Schmidt MH, Varga PP, et al: Reliability analysis of the epidural spinal cord compression scale. J Neurosurg Spine 13:324-328, 2010

12. Buerba RA, Fu MC, Grauer JN: Anterior and posterior cervical fusion in patients with high body mass index are not associated with greater complications. Spine J 14:16431653,2014

13. Bydon A, Xu R, Parker SL, McGirt MJ, Bydon M, Gokaslan ZL, et al: Recurrent back and leg pain and cyst reformation after surgical resection of spinal synovial cysts: systematic review of reported postoperative outcomes. Spine J 10:820-826, 2010

14. Bydon M, Abt NB, De la Garza-Ramos R, Macki M, Witham TF, Gokaslan ZL, et al: Impact of resident participation on morbidity and mortality in neurosurgical procedures: an analysis of 16,098 patients. J Neurosurg 122:955-961, 2015

15. Bydon M, Abt NB, Macki M, Brem H, Huang J, Bydon A, et al: Preoperative anemia increases postoperative morbidity in elective cranial neurosurgery. Surg Neurol Int 5:156, 2014

16. Bydon M, De la Garza-Ramos R, Suk I, McCarthy E, Yamada Y, Wolinsky JP, et al: Single-staged multilevel spondylectomy for en bloc resection of an epithelioid sarcoma with intradural extension in the cervical spine: technical case report. Neurosurgery [epub ahead of print], 2015

17. Bydon M, Gokaslan ZL: Spinal meningioma resection. World Neurosurg 83:1032-1033, 2015

18. Bydon M, Ma TM, Xu R, Weingart J, Olivi A, Gokaslan ZL, et al: Surgical outcomes of craniocervial junction meningiomas: a series of 22 consecutive patients. Clin Neurol Neurosurg 117:71-79, 2014 
19. Chaichana KL, Pendleton C, Sciubba DM, Wolinsky JP, Gokaslan ZL: Outcome following decompressive surgery for different histological types of metastatic tumors causing epidural spinal cord compression. Clinical article. J Neurosurg Spine 11:56-63, 2009

20. Chamberlain MC, Tredway TL: Adult primary intradural spinal cord tumors: a review. Curr Neurol Neurosci Rep 11:320-328, 2011

21. Chi JH, Acosta FL Jr, Aryan HE, Chou D, Ames CP: Partial spondylectomy: modification for lateralized malignant spinal column tumors of the cervical or lumbosacral spine. $\mathbf{J}$ Clin Neurosci 15:43-48, 2008

22. Chi JH, Bydon A, Hsieh P, Witham T, Wolinsky JP, Gokaslan ZL: Epidemiology and demographics for primary vertebral tumors. Neurosurg Clin N Am 19:1-4, 2008

23. Chi JH, Parsa AT: Intramedullary spinal cord metastasis: clinical management and surgical considerations. Neurosurg Clin N Am 17:45-50, 2006

24. Chi JH, Sciubba DM, Rhines LD, Gokaslan ZL: Surgery for primary vertebral tumors: en bloc versus intralesional resection. Neurosurg Clin N Am 19:111-117, 2008

25. Choy W, Lam SK, Smith ZA, Dahdaleh NS: Predictors of thirty day hospital readmission following posterior cervical fusion in 3401 patients. Spine (Phila Pa 1976) [epub ahead of print], 2016

26. Clarke MJ, Dasenbrock H, Bydon A, Sciubba DM, McGirt MJ, Hsieh PC, et al: Posterior-only approach for en bloc sacrectomy: clinical outcomes in 36 consecutive patients. Neurosurgery 71:357-364, 2012

27. Dasenbrock HH, Clarke MJ, Thompson RE, Gokaslan ZL, Bydon A: The impact of July hospital admission on outcome after surgery for spinal metastases at academic medical centers in the United States, 2005 to 2008. Cancer 118:1429-1438, 2012

28. Dasenbrock HH, Devine CA, Liu KX, Gormley WB, Claus EB, Smith TR, et al: Thrombocytopenia and craniotomy for tumor: A National Surgical Quality Improvement Program analysis. Cancer 122:1708-1717, 2016

29. Dasenbrock HH, Liu KX, Devine CA, Chavakula V, Smith TR, Gormley WB, et al: Length of hospital stay after craniotomy for tumor: a National Surgical Quality Improvement Program analysis. Neurosurg Focus 39(6):E12, 2015

30. Dasenbrock HH, Pradilla G, Witham TF, Gokaslan ZL, Bydon A: The impact of weekend hospital admission on the timing of intervention and outcomes after surgery for spinal metastases. Neurosurgery 70:586-593, 2012

31. Dasenbrock HH, Wolinsky JP, Sciubba DM, Witham TF, Gokaslan ZL, Bydon A: The impact of insurance status on outcomes after surgery for spinal metastases. Cancer 118:4833-4841, 2012

32. Dea N, Versteeg A, Fisher C, Kelly A, Hartig D, Boyd M, et al: Adverse events in emergency oncological spine surgery: a prospective analysis. J Neurosurg Spine 21:698-703, 2014

33. Duong LM, McCarthy BJ, McLendon RE, Dolecek TA, Kruchko C, Douglas LL, et al: Descriptive epidemiology of malignant and nonmalignant primary spinal cord, spinal meninges, and cauda equina tumors, United States, 20042007. Cancer 118:4220-4227, 2012

34. Fehlings MG, Nater A, Zamorano JJ, Tetreault LA, Varga PP, Gokaslan ZL, et al: Risk factors for recurrence of surgically treated conventional spinal schwannomas: analysis of 169 patients from a multicenter international database. Spine (Phila Pa 1976) 41:390-398, 2016

35. Fisher CG, Versteeg AL, Dea N, Boriani S, Varga PP, Dekutoski MB, et al: Surgical management of spinal chondrosarcomas. Spine (Phila Pa 1976) 41:678-685, 2016

36. Fourney DR, Gokaslan ZL: Current management of sacral chordoma. Neurosurg Focus 15(2):E9, 2003
37. Fourney DR, Rhines LD, Hentschel SJ, Skibber JM, Wolinsky JP, Weber KL, et al: En bloc resection of primary sacral tumors: classification of surgical approaches and outcome. J Neurosurg Spine 3:111-122, 2005

38. Ghostine S, Vaynman S, Schoeb JS, Cambron H, King WA, Samudrala S, et al: Image-guided thoracoscopic resection of thoracic dumbbell nerve sheath tumors. Neurosurgery 70:461-468, 2012

39. Goldstein CL, Varga PP, Gokaslan ZL, Boriani S, Luzzati A, Rhines L, et al: Spinal hemangiomas: results of surgical management for local recurrence and mortality in a multicenter study. Spine (Phila Pa 1976) 40:656-664, 2015

40. Golinvaux NS, Bohl DD, Basques BA, Fu MC, Gardner EC, Grauer JN: Limitations of administrative databases in spine research: a study in obesity. Spine J 14:2923-2928, 2014

41. Gologorsky Y, Knightly JJ, Chi JH, Groff MW: The Nationwide Inpatient Sample database does not accurately reflect surgical indications for fusion. J Neurosurg Spine 21:984-993, 2014

42. Goodwin CR, Khattab MH, Sankey EW, Elder BD, Kosztowski TA, Sarabia-Estrada R, et al: Factors associated with life expectancy in patients with metastatic spine disease from adenocarcinoma of the lung. Global Spine J 5:417424, 2015

43. Guppy KH, Brara HS, Bernbeck JA: Occipitocervical fusions in elderly patients: mortality and reoperation rates from a national spine registry. World Neurosurg 86:161167,2016

44. Harrop JS, Ganju A, Groff M, Bilsky M: Primary intramedullary tumors of the spinal cord. Spine (Phila Pa 1976) 34 (22 Suppl):S69-S77, 2009

45. Hein PN, Lieber B, Bruce E, Taylor B, Appelboom G, Abraham M, et al: Influence on morbidity and mortality of neoadjuvant radiation and chemotherapy among cranial malignancy patients in the postoperative setting. J Clin Neurosci 22:998-1001, 2015

46. Holman PJ, Suki D, McCutcheon I, Wolinsky JP, Rhines LD, Gokaslan ZL: Surgical management of metastatic disease of the lumbar spine: experience with 139 patients. J Neurosurg Spine 2:550-563, 2005

47. Hsu W, Kosztowski TA, Zaidi HA, Dorsi M, Gokaslan ZL, Wolinsky JP: Multidisciplinary management of primary tumors of the vertebral column. Curr Treat Options Oncol 10:107-125, 2009

48. Jacobs WB, Perrin RG: Evaluation and treatment of spinal metastases: an overview. Neurosurg Focus 11(6):e10, 2001

49. Jalai CM, Worley N, Poorman GW, Cruz DL, Vira S, Passias PG: Surgical site infections following operative management of cervical spondylotic myelopathy: prevalence, predictors of occurrence, and influence on peri-operative outcomes. Eur Spine J 25:1891-1896, 2016

50. Ju DG, Zadnik PL, Groves ML, Hwang L, Kaloostian PE, Wolinksy JP, et al: Factors associated with improved outcomes following decompressive surgery for prostate cancer metastatic to the spine. Neurosurgery 73:657-666, 2013

51. Kaloostian PE, Yurter A, Zadnik PL, Sciubba DM, Gokaslan ZL: Current paradigms for metastatic spinal disease: an evidence-based review. Ann Surg Oncol 21:248-262, 2014

52. Kaloostian PE, Zadnik PL, Etame AB, Vrionis FD, Gokaslan ZL, Sciubba DM: Surgical management of primary and metastatic spinal tumors. Cancer Contr 21:133-139, 2014

53. Kim BD, Smith TR, Lim S, Cybulski GR, Kim JY: Predictors of unplanned readmission in patients undergoing lumbar decompression: multi-institutional analysis of 7016 patients. J Neurosurg Spine 20:606-616, 2014

54. Kimmell KT, Walter KA: Risk factors for venous thromboembolism in patients undergoing craniotomy for neoplastic disease. J Neurooncol 120:567-573, 2014 
55. Kukreja S, Kalakoti P, Ahmed O, Nanda A: Predictors of reoperation-free survival following decompression-alone lumbar spine surgery for on-the-job injuries. Clin Neurol Neurosurg 135:41-45, 2015

56. Lam TC, Uno H, Krishnan M, Lutz S, Groff M, Cheney M, et al: Adverse outcomes after palliative radiation therapy for uncomplicated spine metastases: role of spinal instability and single-fraction radiation therapy. Int $\mathbf{J}$ Radiat Oncol Biol Phys 93:373-381, 2015

57. Lukasiewicz AM, Grant RA, Basques BA, Webb ML, Samuel AM, Grauer JN: Patient factors associated with 30-day morbidity, mortality, and length of stay after surgery for subdural hematoma: a study of the American College of Surgeons National Surgical Quality Improvement Program. J Neurosurg 124:760-766, 2016

58. Mahadevan A, Floyd S, Wong E, Jeyapalan S, Groff M, Kasper E: Stereotactic body radiotherapy reirradiation for recurrent epidural spinal metastases. Int J Radiat Oncol Biol Phys 81:1500-1505, 2011

59. Manoharan SR, Baker DK, Pasara SM, Ponce B, Deinlein D, Theiss SM: Thirty-day readmissions following adult spinal deformity surgery: an analysis of the National Surgical Quality Improvement Program (NSQIP) database. Spine J [epub ahead of print], 2016

60. Martin CT, Pugely AJ, Gao Y, Ilgenfritz RM, Weinstein SL: Incidence and risk factors for early wound complications after spinal arthrodesis in children: analysis of 30-day follow-up data from the ACS-NSQIP. Spine (Phila Pa 1976) 39:1463-1470, 2014

61. McCutcheon BA, Ciacci JD, Marcus LP, Noorbakhsh A, Gonda DD, McCafferty R, et al: Thirty-day perioperative outcomes in spinal fusion by specialty within the NSQIP database. Spine (Phila Pa 1976) 40:1122-1131, 2015

62. McPherson CM, Suki D, McCutcheon IE, Gokaslan ZL, Rhines LD, Mendel E: Metastatic disease from spinal chordoma: a 10-year experience. J Neurosurg Spine 5:277280, 2006

63. Medin PM, Solberg TD, De Salles AA, Cagnon CH, Selch MT, Johnson JP, et al: Investigations of a minimally invasive method for treatment of spinal malignancies with LINAC stereotactic radiation therapy: accuracy and animal studies. Int J Radiat Oncol Biol Phys 52:1111-1122, 2002

64. Merkow RP, Bilimoria KY, McCarter MD, Cohen ME, Barnett CC, Raval MV, et al: Post-discharge venous thromboembolism after cancer surgery: extending the case for extended prophylaxis. Ann Surg 254:131-137, 2011

65. Mesfin A, Buchowski JM, Gokaslan ZL, Bird JE: Management of metastatic cervical spine tumors. J Am Acad Orthop Surg 23:38-46, 2015

66. Moussazadeh N, Laufer I, Yamada Y, Bilsky MH: Separation surgery for spinal metastases: effect of spinal radiosurgery on surgical treatment goals. Cancer Contr 21:168-174, 2014

67. Mukherjee D, Chaichana KL, Gokaslan ZL, Aaronson O, Cheng JS, McGirt MJ: Survival of patients with malignant primary osseous spinal neoplasms: results from the Surveillance, Epidemiology, and End Results (SEER) database from 1973 to 2003. J Neurosurg Spine 14:143-150, 2011

68. Mukherjee D, Chaichana KL, Parker SL, Gokaslan ZL, McGirt MJ: Association of surgical resection and survival in patients with malignant primary osseous spinal neoplasms from the Surveillance, Epidemiology, and End Results (SEER) database. Eur Spine J 22:1375-1382, 2013

69. Patchell RA, Tibbs PA, Regine WF, Payne R, Saris S, Kryscio RJ, et al: Direct decompressive surgical resection in the treatment of spinal cord compression caused by metastatic cancer: a randomised trial. Lancet 366:643-648, 2005

70. Patel NP, Wolcott WP, Johnson JP, Cambron H, Lewin M,
McBride D, et al: Esophageal injury associated with anterior cervical spine surgery. Surg Neurol 69:20-24, 2008

71. Pugely AJ, Martin CT, Gao Y, Ilgenfritz R, Weinstein SL: The incidence and risk factors for short-term morbidity and mortality in pediatric deformity spinal surgery: an analysis of the NSQIP pediatric database. Spine (Phila Pa 1976) 39:1225-1234, 2014

72. Quraishi NA, Rajabian A, Spencer A, Arealis G, Mehdian H, Boszczyk BM, et al: Reoperation rates in the surgical treatment of spinal metastases. Spine J 15 (3 Suppl):S37-S43, 2015

73. Sato S, Yagi M, Machida M, Yasuda A, Konomi T, Miyake A, et al: Reoperation rate and risk factors of elective spinal surgery for degenerative spondylolisthesis: minimum 5-year follow-up. Spine J 15:1536-1544, 2015

74. Schairer WW, Carrer A, Sing DC, Chou D, Mummaneni PV, Hu SS, et al: Hospital readmission rates after surgical treatment of primary and metastatic tumors of the spine. Spine (Phila Pa 1976) 39:1801-1808, 2014

75. Scheer JK, Tang JA, Smith JS, Klineberg E, Hart RA, Mundis GM Jr, et al: Reoperation rates and impact on outcome in a large, prospective, multicenter, adult spinal deformity database: clinical article. J Neurosurg Spine (Phila Pa 1976) 19:464-470, 2013

76. Schmeler KM, Wilson GL, Cain K, Munsell MF, Ramirez PT, Soliman PT, et al: Venous thromboembolism (VTE) rates following the implementation of extended duration prophylaxis for patients undergoing surgery for gynecologic malignancies. Gynecol Oncol 128:204-208, 2013

77. Schoenfeld AJ, Le HV, Marjoua Y, Leonard DA, Belmont PJ Jr, Bono CM, et al: Assessing the utility of a clinical prediction score regarding 30-day morbidity and mortality following metastatic spinal surgery: the New England Spinal Metastasis Score (NESMS). Spine J 16:482-490, 2016

78. Sciubba DM, Macki M, Bydon M, Germscheid NM, Wolinsky JP, Boriani S, et al: Long-term outcomes in primary spinal osteochondroma: a multicenter study of 27 patients. J Neurosurg Spine 22:582-588, 2015

79. Sciubba DM, Nelson C, Gok B, McGirt MJ, McLoughlin GS, Noggle JC, et al: Evaluation of factors associated with postoperative infection following sacral tumor resection. J Neurosurg Spine 9:593-599, 2008

80. Sciubba DM, Petteys RJ, Dekutoski MB, Fisher CG, Fehlings MG, Ondra SL, et al: Diagnosis and management of metastatic spine disease. A review. J Neurosurg Spine 13:94-108, 2010

81. Sciubba DM, Petteys RJ, Garces-Ambrossi GL, Noggle JC, McGirt MJ, Wolinsky JP, et al: Diagnosis and management of sacral tumors. J Neurosurg Spine 10:244-256, 2009

82. Sebastian A, Huddleston P III, Kakar S, Habermann E, Wagie A, Nassr A: Risk factors for surgical site infection after posterior cervical spine surgery: an analysis of 5441 patients from the ACS-NSQIP 2005-2012. Spine J 16:504509, 2016

83. Seicean A, Seicean S, Schiltz NK, Alan N, Jones PK, Neuhauser D, et al: Short-term outcomes of craniotomy for malignant brain tumors in the elderly. Cancer 119:10581064, 2013

84. Sellers MM, Merkow RP, Halverson A, Hinami K, Kelz RR, Bentrem DJ, et al: Validation of new readmission data in the American College of Surgeons National Surgical Quality Improvement Program. J Am Coll Surg 216:420-427, 2013

85. Sharma M, Sonig A, Ambekar S, Nanda A: Discharge dispositions, complications, and costs of hospitalization in spinal cord tumor surgery: analysis of data from the United States Nationwide Inpatient Sample, 2003-2010. J Neurosurg Spine 20:125-141, 2014

86. Shehadi JA, Sciubba DM, Suk I, Suki D, Maldaun MV, McCutcheon IE, et al: Surgical treatment strategies and out- 
come in patients with breast cancer metastatic to the spine: a review of 87 patients. Eur Spine J 16:1179-1192, 2007

87. Shirzadi A, Drazin D, Gates M, Shirzadi N, Bannykh S, Fan $\mathrm{X}$, et al: Surgical management of primary spinal hemangiopericytomas: an institutional case series and review of the literature. Eur Spine J 22 (Suppl 3):S450-S459, 2013 [Erratum in Eur Spine J 22 (Suppl 3):S460, 2013]

88. Shirzadi AS, Drazin DG, Strickland AS, Bannykh SI, Johnson JP: Vertebral column metastases from an esthesioneuroblastoma: chemotherapy, radiation, and resection for recurrence with 15-year followup. Case Rep Surg 2013: 107315,2013

89. Singh DC, Yue JK, Metz LN, Winkler EA, Zhang WR, Burch S, et al: Obesity is an independent risk factor of early complications after revision spine surgery. Spine (Phila Pa 1976) 41:E632-E640, 2016

90. Sippel RS, Chen H: Limitations of the ACS NSQIP in thyroid surgery. Ann Surg Oncol 18:3529-3530, 2011

91. Su AW, Habermann EB, Thomsen KM, Milbrandt TA, Nassr A, Larson AN: Risk factors for 30-day unplanned readmission and major perioperative complications following spine fusion surgery in adults: a review of the National Surgical Quality Improvement Program (NSQIP) database. Spine (Phila Pa 1976) [epub ahead of print], 2016

92. Varga PP, Szövérfi Z, Fisher CG, Boriani S, Gokaslan ZL, Dekutoski MB, et al: Surgical treatment of sacral chordoma: prognostic variables for local recurrence and overall survival. Eur Spine J 24:1092-1101, 2015

93. Villavicencio AT, Oskouian RJ, Roberson C, Stokes J, Park J, Shaffrey CI, et al: Thoracolumbar vertebral reconstruction after surgery for metastatic spinal tumors: long-term outcomes. Neurosurg Focus 19(3):E8, 2005

94. Wang MC, Shivakoti M, Sparapani RA, Guo C, Laud PW, Nattinger AB: Thirty-day readmissions after elective spine surgery for degenerative conditions among US Medicare beneficiaries. Spine J 12:902-911, 2012

95. Williams BJ, Fox BD, Sciubba DM, Suki D, Tu SM, Kuban D, et al: Surgical management of prostate cancer metastatic to the spine. J Neurosurg Spine 10:414-422, 2009

96. Witham TF, Khavkin YA, Gallia GL, Wolinsky JP, Gokaslan ZL: Surgery insight: current management of epidural spinal cord compression from metastatic spine disease. Nat Clin Pract Neurol 2:87-94, 116, 2006

97. Xu R, McGirt MJ, Parker SL, Bydon M, Olivi A, Wolinsky $\mathrm{JP}$, et al: Factors associated with recurrent back pain and cyst recurrence after surgical resection of one hundred ninety-five spinal synovial cysts: analysis of one hundred sixty-seven consecutive cases. Spine (Phila Pa 1976) 35:1044-1053, 2010
98. Yao KC, Boriani S, Gokaslan ZL, Sundaresan N: En bloc spondylectomy for spinal metastases: a review of techniques. Neurosurg Focus 15(5):E6, 2003

99. York JE, Berk RH, Fuller GN, Rao JS, Abi-Said D, Wildrick DM, et al: Chondrosarcoma of the spine: 1954 to 1997. J Neurosurg 90 (1 Suppl):73-78, 1999

100. Zadnik PL, Goodwin CR, Karami KJ, Mehta AI, Amin AG, Groves ML, et al: Outcomes following surgical intervention for impending and gross instability caused by multiple myeloma in the spinal column. J Neurosurg Spine 22:301309,2015

\section{Disclosures}

Dr. Groff has served as a consultant to DePuy Spine and Biomet Spine, and has received development royalties from Biomet Spine. Dr. Codman has served as a consultant to Covidien and Proctor. Dr. Grof has served as a consultant to DePuy Synthes, and has ownership in Biomet Spine. Dr. Chi has served as a consultant to DePuy Synthes Spine. The ACS NSQIP and the hospitals participating in the ACS NSQIP are the source of the data used herein; they have not verified and are not responsible for the statistical validity of the data analysis or the conclusions derived by the authors.

\section{Author Contributions}

Conception and design: Smith, Karhade, Vasudeva. Acquisition of data: Karhade, Dasenbrock. Analysis and interpretation of data: Karhade, Vasudeva, Dasenbrock. Drafting the article: Karhade, Vasudeva, Dasenbrock. Critically revising the article: Karhade, Vasudeva, Dasenbrock, Chi. Reviewed submitted version of manuscript: all authors. Approved the final version of the manuscript on behalf of all authors: Smith. Statistical analysis: Smith, Karhade, Dasenbrock. Administrative/technical/material support: Lu, Gormley, Groff, Chi. Study supervision: Smith, Lu, Gormley, Groff, Chi.

\section{Supplemental Information Videos \\ Video Abstract. https://vimeo.com/173610430.}

\section{Correspondence}

Timothy R. Smith, Department of Neurosurgery, Brigham and Women's Hospital and Harvard Medical School, 75 Francis St., Boston, MA 02115. email: trsmith@partners.org. 